\title{
Addendum to "Causality, Kramers-Kronig Relations, and Landau Damping" [The Open Plasma Physics Journal, 2012, 5, 36-40]
}

\author{
Hee J. Lee*
}

Department of Physics, Hanyang University, Seoul 133-791, Korea

\begin{abstract}
It is shown that the Landau contour can be derived directly from the causality requirement. The KramersKronig relations are derived directly from the causal requirement without using analytic continuation.
\end{abstract}

Keywords: Causality, Kramers-Kronig Relations, Landau damping.

In earlier paper [1], it was shown that the plasma susceptibility evaluated along the Landau contour [2] is equivalent to the Kramers-Kronig relations and therefore it was concluded that the collisionless damping of plasma waves can be traced to the causality. In this addendum to ref. [1], it is shown that the collisionless damping can be derived formally from the causality requirement. The key element leading to this conclusion is to fully enumerate the singular algebraic expression $\frac{1}{\omega-k v}$.

We begin with the expression for the susceptibility $\chi(k, \omega)$ of the electron plasma wave [1]

$\chi(k, \omega)=\frac{\omega_{p e}^{2}}{k} \int_{-\infty}^{\infty} d v \frac{d g_{0}}{d v} \frac{1}{\omega-k v}$

where $\omega_{p e}$ is the electron plasma frequency, $g_{0}(v)$ is the zero order equilibrium velocity distribution function. The form of Eq. (1) for the susceptibility can be obtained either kinetically or fluidly [1]. Here we pay special attention to the algebraic expression, $\frac{1}{\omega-k v}$. This algebraic quantity is well defined if $\omega \neq k v$ but it should be more defined when $\omega=k v$ because the denominator gives a singularity. In this case, the expression should be rephrased so that the singularity can be dealt with unequivocally. We use the wellknown identity [3]:

$\frac{1}{\omega-k v}=P \frac{1}{\omega-k v}+\lambda \delta(\omega-k v)$

where the symbol $\mathrm{P}$ denotes the principal value and $\lambda$ is an undetermined constant. Equation (2) is correct because of the two identities:

$(x-a) P \frac{1}{x-a}=1,(x-a) \delta(x-a)=0$

*Address correspondence to this author at the Department of Physics, Hanyang University, Seoul 133-791, Korea; E-mail: ychjlee@yahoo.com
In fact, Eq.(2) was the mathematical motivation of the Van Kampen modes of plasma wave [3]. Then, Eq. (1) is rewritten

$\chi(k, \omega)=\frac{\omega_{p e}^{2}}{k} \int_{-\infty}^{\infty} d v \frac{d g_{0}}{d v}\left[P \frac{1}{\omega-k v}+\lambda \delta(\omega-k v)\right]$

The constant $\lambda$ will be determined by satisfying the causal requirement $[1,4]$ :

$\chi(t)=0$ for $t<0$

Let us invert Eq.(3):

$\chi(k, t)=\int_{-\infty}^{\infty} \chi(k, \omega) e^{-i \omega t} \frac{d \omega}{2 \pi}$

$=\frac{\omega_{p e}^{2}}{2 \pi k} \int_{-\infty}^{\infty} d v \frac{d g_{0}}{d v} e^{-i k v t}\left[P \int_{-\infty}^{\infty} d \omega \frac{e^{-i \omega t}}{\omega}+\lambda\right]$

The principal part integral is the step function:

$P \int_{-\infty}^{\infty} \frac{e^{-i x t}}{x} d x=i \pi(t<0)$ or $-i \pi(t>0)$

The causal requirement in Eq. (4) is satisfied by $\lambda=-i \pi$, and therefore the plasma susceptibility in Eq. (3) becomes

$\chi(k, \omega)=\frac{\omega_{p e}^{2}}{k} \int_{-\infty}^{\infty} d v \frac{d g_{0}}{d v}\left[P \frac{1}{\omega-k v}-i \pi \delta(\omega-k v)\right]$

which agrees with the susceptibility evaluated along the Landau contour.

In earlier work [1], it was shown that Eq. (7) is compatible with the Kramers-Kronig (K-K) relations, and the imaginary (real) part of $\chi(\omega)$ can be derived from the real (imaginary) part of $\chi(\omega)$. Here we present a new and more economic way of deriving the $\mathrm{K}-\mathrm{K}$ relations by applying the constraint on $\chi(\omega)$ in Eq. (4). One can enforce the constraint in Eq. (4) by writing

$\chi(t)=\frac{1}{2}[S(t)+1] \chi(t)$ 
where $S(t)$ is the step function: $\mathrm{S}(\mathrm{t})=1$ for $t>0$ and $S(t)=-1$ for $t<0$, and the $k$-dependance is implicit. Clearly, the right hand side of Eq. (8) is equal to $\chi(t)$ for $t>0$ and zero for $t<0$. Fourier transform of Eq. (8) is written as a convolution integral

$\chi(\omega)=\frac{1}{2} \frac{1}{2 \pi} \int_{-\infty}^{\infty} d \omega^{\prime} \chi\left(\omega^{\prime}\right) S\left(\omega-\omega^{\prime}\right)+\frac{1}{2} \chi(\omega)$

The Fourier transform of the step function is

$S(\omega)=2 i P \frac{1}{\omega}$

Using Eq. (10) in Eq. (9) yields

$\chi(\omega)=\frac{i}{\pi} P \int_{-\infty}^{\infty} \frac{\chi\left(\omega^{\prime}\right)}{\omega-\omega^{\prime}} d \omega^{\prime}$

This is the implicit form of the $\mathrm{K}-\mathrm{K}$ relations obtained by direct application of the causal requirement in Eq. (4). Writing $\chi=\operatorname{Re} \chi+i \operatorname{Im} \chi$ and separating the real and the imaginary parts, Eq. (11) gives the K-K relations

$$
\begin{aligned}
& \operatorname{Re} \chi(\omega)=-\frac{1}{\pi} P \int_{-\infty}^{\infty} \frac{\operatorname{Im} \chi\left(\omega^{\prime}\right)}{\omega-\omega^{\prime}} d \omega^{\prime} \\
& \operatorname{Im} \chi(\omega)=\frac{1}{\pi} P \int_{-\infty}^{\infty} \frac{\operatorname{Re} \chi\left(\omega^{\prime}\right)}{\omega-\omega^{\prime}} d \omega^{\prime}
\end{aligned}
$$

As a consistency check, we determine the constant $\lambda$ in Eq. (3) by using Eq. (11). Substituting Eq. (3) into Eq. (11) gives

$$
\chi(\omega)=\frac{i}{\pi} \frac{\omega_{p e}^{2}}{k} \int_{-\infty}^{\infty} d v \frac{d g_{0}}{d v} P \int_{-\infty}^{\infty} \frac{d \omega^{\prime}}{\omega-\omega^{\prime}}\left[P \frac{1}{\omega^{\prime}-k v}+\lambda \delta\left(\omega^{\prime}-k v\right)\right]
$$

Upon using the formula
$P \int_{-\infty}^{\infty} \frac{1}{x^{\prime}-x} \frac{1}{x^{\prime}-x^{\prime \prime}} d x^{\prime}=\pi^{2} \delta\left(x-x^{\prime \prime}\right)$

Equation (12) becomes

$\chi(k, \omega)=\frac{\omega_{p e}^{2}}{k} \int_{-\infty}^{\infty} d v \frac{d g_{0}}{d v}\left[-i \pi \delta(\omega-k v)+\frac{i \lambda}{\pi} P \frac{1}{\omega-k v}\right]$

Equations (3) and (13) should read as the same equation by appropriate choice of $\lambda$. By inspection, we find that $\lambda$ should be equal to $-i \pi$, and Eq. (13) becomes Eq. (7).

We have shown that the imaginary part of the plasma susceptibility can be derived from the causal constraint in Eq. (4), thus showing directly the connection of the collisionless damping with the causality. The derivation of the Kramers-Kronig relations from the causal constraint in Eq. (4) appears to be new, to the author's knowledge, and is economic in as much as we did not use the analytic continuation of the function $\chi(\omega)$.

\section{CONFLICT OF INTEREST}

The author confirms that this article content has no conflict of interest.

\section{ACKNOWLEDGEMENTS}

Declared none.

\section{REFERENCES}

[1] Lim YK, Hee JL. Causality, Kramers-Kronig relations, and Landau damping. Open Plasma Phys J 2012; 5: 36-43.

[2] Landau L. On the vibration of the electronic plasma. J Phys 1946; 10: 25-34.

[3] Van Kampen NG, Felderhof BU. Theoretical methods in plasma physics. New York: John Wiley 1967.

[4] Jackson JD. Classical electrodynamics. Chapter 7. New York: John Wiley 1974. 